\title{
Novel Hybrid Mesh Surgery Combines Sacrocolpopexy with Transvaginal Mesh Placement for Pelvic Organ Prolapse
}

\author{
Masao Ichikawa, Shigeo Akira, Katsuya Mine, Nozomi Ohuchi, \\ Nao Iwasaki, Keisuke Kurose and Toshiyuki Takeshita \\ Division of Reproductive Medicine, Perinatology and Gynecologic Oncology, Graduate School of Medicine, Nippon Medical School \\ Department of Obstetrics and Gynecology, Nippon Medical School
}

\begin{abstract}
Mesh surgeries, such as sacrocolpopexy and transvaginal mesh surgery, are commonly used to treat pelvic organ prolapse. Although mesh surgeries have a high success rate, they are unsuitable for some patients. For a patient with pelvic organ prolapse and highly calcified multiple fibroids, we performed hybrid sacrocolpopexy combined with transvaginal mesh surgery with a method modified for the patient's condition. Three months after surgery, the results were highly satisfactory. This approach is simple, secure, and versatile for patients who are not good candidates for conventional mesh surgeries. This novel hybrid mesh surgery is an option for treating various types of pelvic organ prolapse.
\end{abstract}

(J Nippon Med Sch 2011; 78: 379-383)

Key words: pelvic organ prolapse, sacrocolpopexy, transvaginal mesh, cystocele, paravaginal defect, fibroids

\section{Introduction}

Pelvic organ prolapse (POP) is caused by multicompartment failures of pelvic support and often coexists with other gynecological conditions, such as fibroids and ovarian tumors. Because the pathologic mechanism of POP is complex, for optimal results some patients require surgeries modified for their individual conditions. In the present case, we treated a patient who had POP and multiple stiff, calcified fibroids. Because her uterine cervix did not descend far enough for an anterior mesh to be tacked to it via a vaginal route in transvaginal mesh (TVM) surgery ${ }^{1}$, TVM was considered unsuitable.
Therefore, we performed abdominal sacrocolpopexy with 2 separate meshes ${ }^{2}$ and concomitant supracervical hysterectomy to remove the multiple stiff, calcified fibroids. In addition, we modified the shape of the anterior mesh used in this surgery into an inverted $\mathrm{T}$ shape to reinforce paravaginal defects. The mesh was initially delivered vaginally to the vesicovaginal space, similarly as in TVM surgery, and was then suspended from the sacral promontory via an abdominal route in the same fashion as in sacrocolpopexy. Hence, we named this novel procedure "hybrid sacrocolpopexy" and report the details of this procedure herein.

Correspondence to Masao Ichikawa, MD, PhD, Department of Obstetrics and Gynecology, Nippon Medical School, 11-5 Sendagi, Bunkyo-ku, Tokyo 113-8603, Japan

E-mail: masai@nms.ac.jp

Journal Website (http://www.nms.ac.jp/jnms/) 


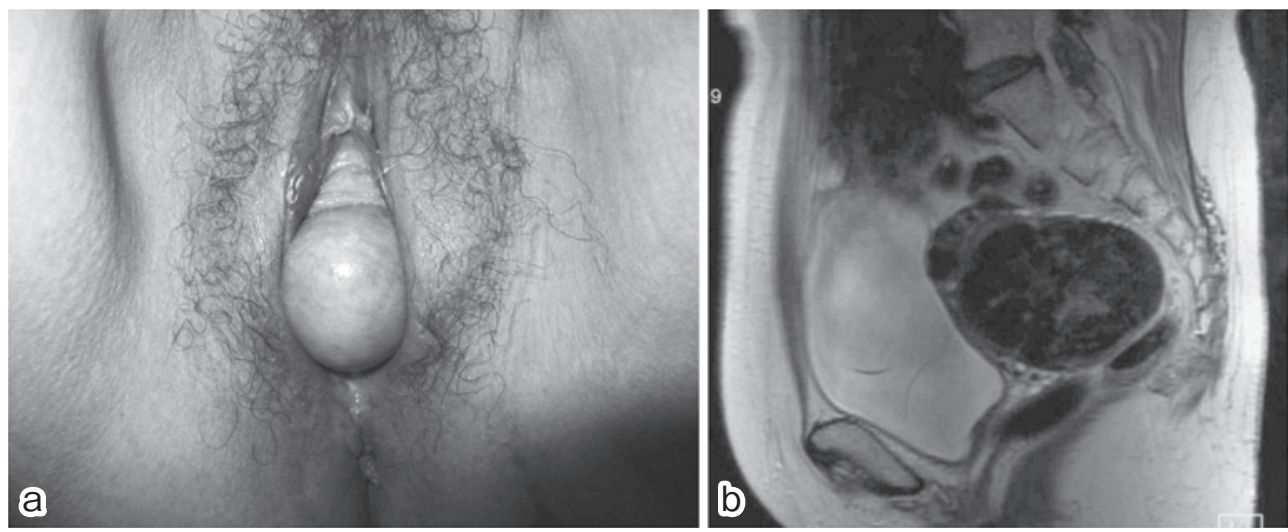

Fig. 1 a: Cystocele $\mathbf{b}$ : MRI of pelvis
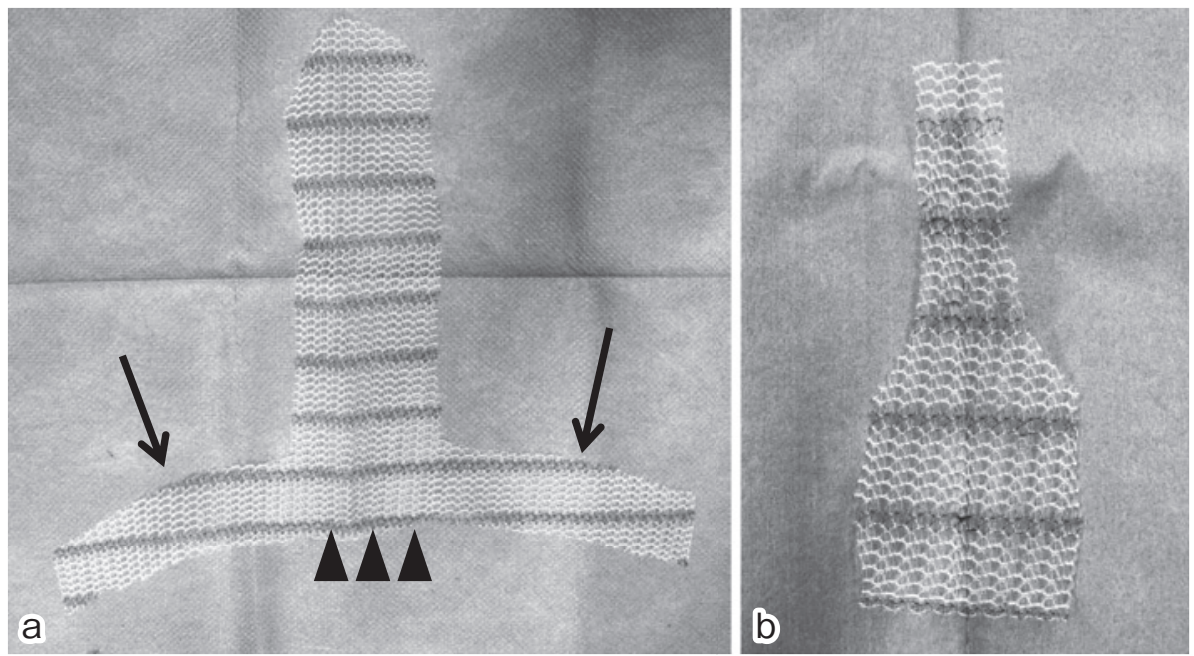

Fig. 2 Meshes

a: Inverted T-shaped anterior mesh. Arrow heads indicates where it is tacked to the vaginal fascia. Arrows indicates the arms which go through obturator fossa. b: Posterior mesh

\section{Case Report}

The patient was 62-year-old woman who had given birth to 2 children. She had a stage III cystocele with a paravaginal defect (Fig. la and Fig. 7), and the uterus contained multiple highly calcified fibroids (Fig. 1b). The surgery began with the patient being place in the lithotomy position with a levitator while under general anesthesia. After water dissection, a median incision was made on the anterior vaginal wall, and the paravaginal space was dissected via a vaginal approach. The central bottom portion of an inverted T-shaped mesh (Fig. 2a, arrow heads) was tacked to the vaginal fascia $3 \mathrm{~cm}$ below the meatus urethra with three 2-0 absorbable sutures (PDS: Ethicon, Somerville, NJ, USA) (Fig. 3a). The arms of the mesh (Fig. 2a, arrows) were inserted through the obturator fossa and were kept tension-free by following the TVM protocol as described by Debodinance et $\mathrm{al}^{1}$ (Fig. 3a, b). Next, the mesh was embedded into the vacancy between the bladder and vagina, and the incision was closed with a continuous 2-0 absorbable suture (Vicryl: Ethicon).

The patient was then placed in the dorsal position. An abdominal median incision was made, and supracervical hysterectomy with concomitant bilateral salpingo-oophorectomy was performed to remove multiple calcified fibroids (Fig. 4a, b). The 

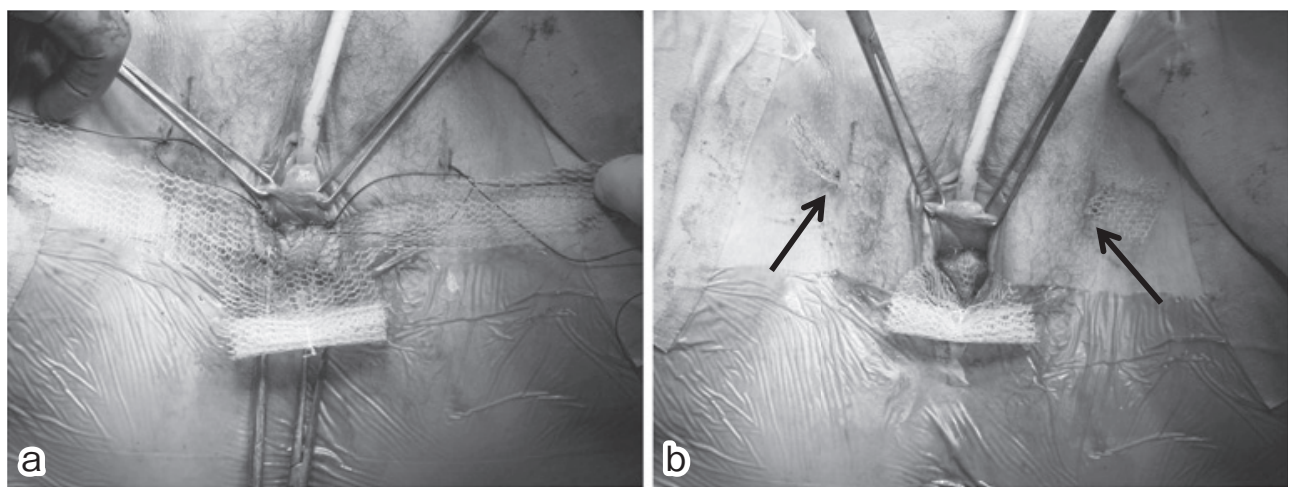

Fig. 3 Procedure of the fixation of the inverted T-shaped anterior mesh

a: The central bottom of the inverted T-shaped mesh was tacked to the vaginal fascia at $3 \mathrm{~cm}$ below the meatus urethra with three 2-0 absorbable sutures. The top portion of the mesh was folded for the embedding. b: Arm parts of the mesh went through the obturator fossa and were kept tension-free (arrows).
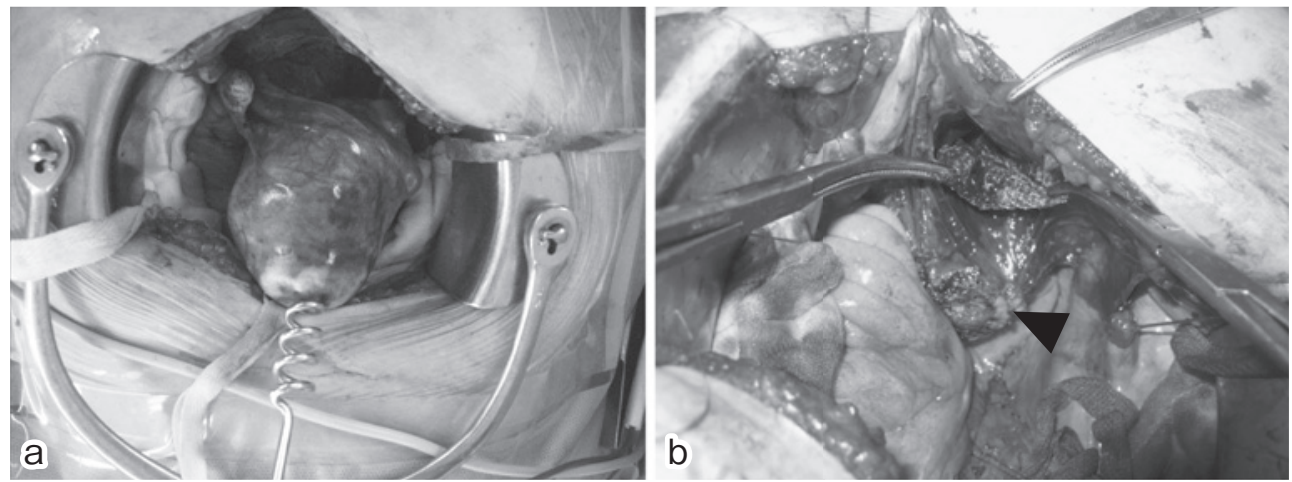

Fig. 4 a: The uterus with calcified multiplefibroids. b: Amputated cervix (arrow head)
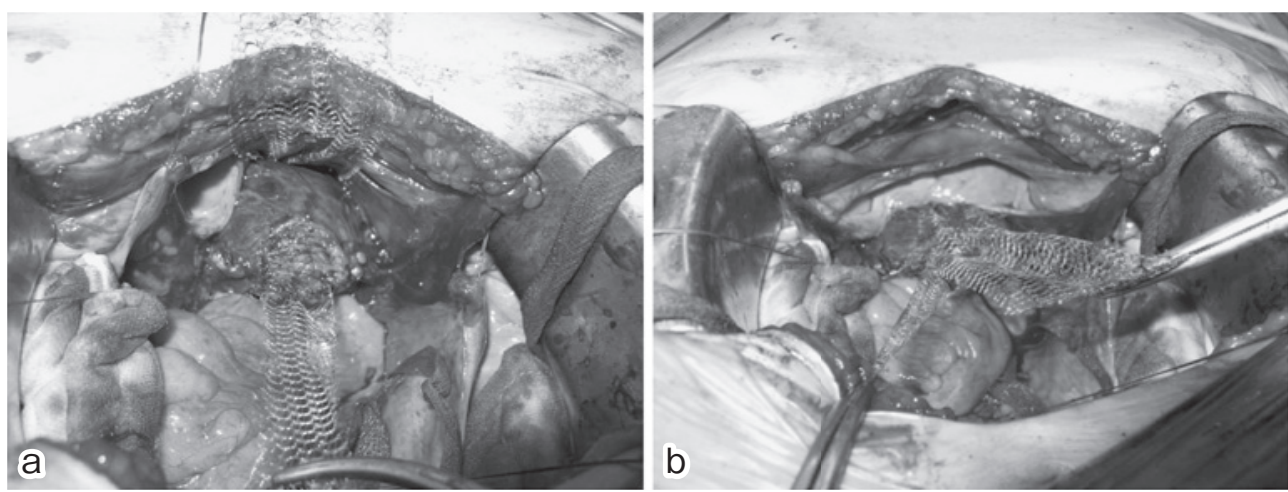

Fig. 5 Fixation of anterior and posterior meshes to the amputated cervix

a: The top of the anterior mesh was pulled up and the middle part of the posterior mesh was tacked to the amputated cervix with three 3-0 non-absorbable sutures. $\mathbf{b}$ : The middle part of the anterior mesh was tacked to the amputated cervix in a similar fashion.

vesicouterine fossa was opened, and the top of the embedded anterior mesh was pulled out into the peritoneal cavity (Fig. $\mathbf{4} \mathbf{b}$ ); the middle part of the mesh was tacked to the amputated cervix with three 3-0 nonabsorbable sutures (Ethibond: Ethicon)
(Fig. 5b). As a result, the anterior mesh was completely attached to the anterior vaginal wall and cervix with 6 sutures: 3 absorbable and 3 nonabsorbable.

Next, the rectouterine pouch was opened and 

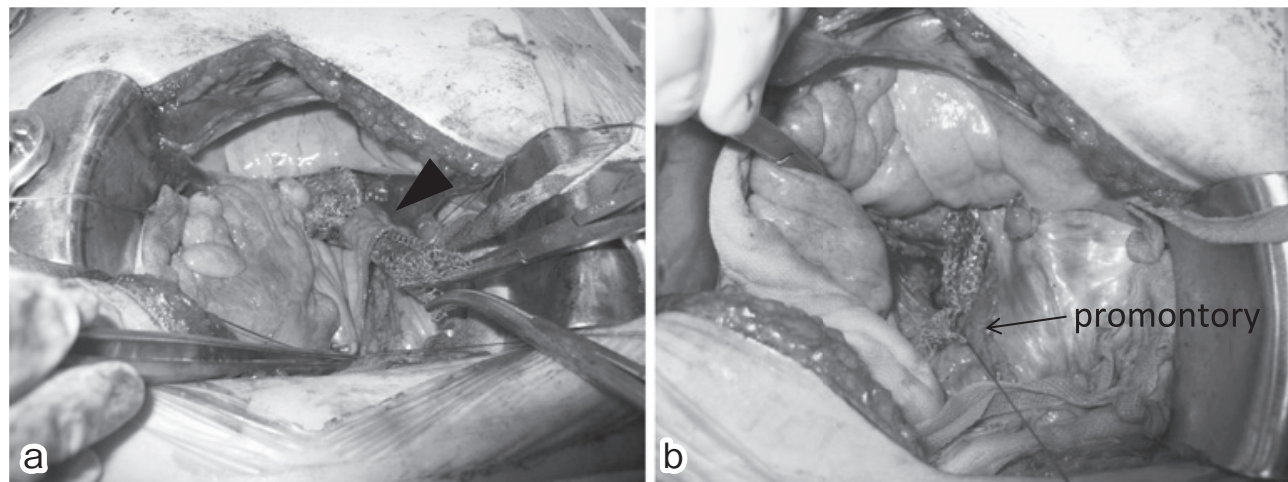

Fig. 6 Fixation of the top of anterior mesh to the sacral promontory

a: The top of the anterior mesh went through the tunnel of retroperitoneum (arrow head). b: Fixation of the top of the anterior mesh to the longitudinal ligament on the sacral promontory with two zero non-absorbable sutures.

\begin{tabular}{|c|c|c|}
\hline+2 & +2 & -5 \\
\hline 4 & 2.5 & 7 \\
\hline-2 & -2 & -7 \\
\hline
\end{tabular}

Pre-surgery

\begin{tabular}{|c|c|c|}
\hline-3 & -3 & -6.5 \\
\hline 2.5 & 2.5 & 7.5 \\
\hline-3 & -3 & -7.5 \\
\hline
\end{tabular}

Three months after surgery

Fig. 7 Pelvic Organ Prolapse Quantification (POPQ)

dissected until half the depth of the posterior vagina, and the bottom portion of a posterior mesh (Fig. $2 \mathbf{b}$ ) was sutured to the posterior vaginal wall with 3 nonabsorbable sutures. The middle part of the posterior mesh was tacked in a similar fashion (Fig. $\mathbf{5 a}, \mathbf{b})$. In short, the posterior mesh was stitched to the posterior vaginal wall with 6 nonabsorbable sutures. Because an obvious rectocele was not present (Fig. 7), the posterior mesh was not fixed to the levator ani.

Finally, the retroperitoneum in front of the sacral promontory was opened, and then the longitudinal ligament at the sacral promontory was visualized. A tunnel was made under the retroperitoneum from the sacral promontory to the Douglas fossa. The top of the anterior mesh was placed through the tunnel and then tacked to the longitudinal ligament on the sacral promontory with 2-0 nonabsorbable sutures (Ethibond: Ethicon) (Fig. 6a, b). The top of the posterior mesh was attached to the retroperitoneum and kept tension-free to avoid bowel problems and dyspareunia. The retroperitoneal space was closed with a 2-0 absorbable suture (Vicryl: Ethicon). Operation time was 232 minutes. Total blood loss was $240 \mathrm{~mL}$. There were no complications during the perioperative period. Pelvic Organ Prolapse Quantification 3 months after surgery showed that the prolapse of the leading point of the anterior vaginal wall had decreased from $+2 \mathrm{~cm}$ to $-3 \mathrm{~cm}$ to the hymen (Fig. 7).

\section{Discussion}

TVM surgery is a superior treatment for POP and is rapidly becoming widespread due to the simplicity of its techniques. On the other hand, reports of severe complications ${ }^{4-7}$ associated with blind puncture, such as urinary trauma and severe bleeding, are also increasing. Therefore, a reevaluation of its safety and a modification of its 
procedure may be necessary. Because second and third punctures for arms are skipped in the form of hybrid sacrocolpopexy discussed in the present study, the risk of complications accompanied with blind punctures can be minimized.

As another approach for POP treatment, abdominal sacrocolpopexy, is a gold standard with a long history of good results in Europe. Although this surgery is effective at providing vertical support (Delancey's level $\mathrm{I}^{8}$ ), the same cannot be said for lateral support (Delancey's level $\mathrm{II}^{8}$ ). As a result, additional paravaginal repair $^{9}$ is concomitantly performed to compensate for the weakness. In our hybrid sacrocolpopexy, the arms of the inverted $\mathrm{T}$ shaped mesh can play a role in paravaginal repair to support paravaginal defects.

Furthermore, TVM preserves the uterus because its concomitant removal increases the risk of mesh exposition from $5 \%$ to $20 \%{ }^{10,11}$. Therefore, patients who require a hysterectomy for large or multiple fibroids are not good candidates for TVM but are candidates for sacrocolpopexy or this type of hybrid sacrocolpopexy, which allows supracervical hysterectomy without an increased rate of mesh exposure $^{11}$. In the present case, open laparotomy was the method chosen for hybrid sacrocolpopexy because of the multiple stiff, calcified fibroids; however, a laparoscopic approach is also possible in normal situations.

This novel approach is technically simple, secure, and versatile to adapt to patients who do are not candidates for conventional TVM or sacrocolpopexy. We believe that this novel method is another option for treatment of various types of POP.

\section{References}

1. Debodinance P, Berrocal J, Clave $\mathrm{H}$, et al:: Changing attitudes on the surgical treatment of urogenital prolapse: birth of the tension-free vaginal mesh. J Gynecol Obstet Biol Reprod (Paris) 2004; 33: 577-588.

2. Gadonneix P, Ercoli A, Salet-Lizee D, et al: Laparoscopic sacrocolpopexy with two separate meshes along the anterior and posterior vaginal walls for multicompartment pelvic organ prolapse. J Am Assoc Gynecol Laparosc 2004; 11: 29-35.

3. Bump RC, Mattiasson A, Bo K, et al.: The standardization of terminology of female pelvic organ prolapse and pelvic floor dysfunction. Am J Obstet Gynecol 1996; 175: 10-17.

4. Feiner B, Jelovsek JE, Maher C: Efficacy and safety of transvaginal mesh kits in the treatment of prolapse of the vaginal apex: a systematic review. BJOG 2009; 116: 15-24.

5. Bekker MD, Bevers RF, Elzevier HW: Transurethral and suprapubic mesh resection after Prolift bladder perforation: a case report. Int Urogynecol J Pelvic Floor Dysfunct 2010; 21: 1301-1303.

6. Gangam N, Kanee A: Retroperitoneal hemorrhage after a vaginal mesh prolapse procedure. Obstet Gynecol 2007; 110 (Pt 2): 463-464.

7. Karateke A, Cam C, Ayaz R: Unilateral hydroureteronephrosis after a mesh procedure. J Minim Invasive Gynecol 2010; 17: 232-234.

8. DeLancey JO: Anatomic aspects of vaginal eversion after hysterectomy. Am J Obstet Gynecol 1992; 166 (Pt 1): 1717-1724; discussion 1724-1718.

9. Maher CF, Feiner B, Decuyper EM, Nichlos CJ, Hickey KV, O’Rourke P: Laparoscopic sacral colpopexy versus total vaginal mesh for vaginal vault prolapse: a randomized trial. Am J Obstet Gynecol 2011; 204: 360 e361-367.

10. Deffieux X, de Tayrac R, Huel C, et al.: Vaginal mesh erosion after transvaginal repaira of cystocele using gynemesh or Gynemesh-soft in 138 women: a comparative study. Int Urogynecol J 2007; 18: 73-79.

11. Tan-Kim J, Menefee SA, Luber KM, Nager CW, Lukacz ES: Prevalence and risk factors for mesh erosion after laparoscopic-assisted sacrocolpopexy. Int Urogynecol J 2011; 22: 205-212.

(Received, May 6, 2011)

(Accepted, May 18, 2011) 